\title{
COVID-19 and the Cytokine Storm
}

\author{
Lawrence 0 Flowers* \\ Biology Department, Livingstone College, United States \\ *Corresponding author: Lawrence O Flowers, Biology Department, Livingstone College, United States
}

\begin{tabular}{|c|}
\hline ARTICLE INFO \\
\hline Received: 蔧 August 08, 2020 \\
\hline Published: August 20, 2020 \\
\hline
\end{tabular}

Citation: Lawrence O Flowers. COVID-19 and the Cytokine Storm. Biomed J Sci \& Tech Res 29(4)-2020. BJSTR. MS.ID.004833.

Keywords: Coronavirus; COVID-19; Cytokines; Inflammation; Cytokine Storm

\section{ABSTRACT}

The World Health Organization (WHO) declared the Coronavirus Disease 2019 (COVID-19), a disease caused by SARS-CoV-2, a pandemic in March 2020 based on the widespread and aggressive nature of the disease. Few, if any, global health crises have affected the human race in the last hundred years like COVID-19. The disease presents many symptoms in patients suggesting that the virus is effective at infecting many different cell types throughout the body. Cytokines are small, low molecular weight proteins produced by immune cells that regulate specific aspects of human immunity. In a virological context, cytokines mediate innate and adaptive immunity in response to viral infections. Recent COVID-19 patient data has shown that immune responses such as the inflammatory response can have positive or negative impacts. Overactivation of cytokine-secreting cells during a systemic inflammatory response often leads to a cytokine storm resulting in an uncontrolled influx of pro-inflammatory mediators in the respiratory tract. Hyperinflammation in the lungs leads to poor pathophysiological outcomes, severe pneumonia, multiorgan failure, and even death in a subset of COVID-19 patients. The utilization of anti-inflammatory therapeutic options to counteract the cytokine storm syndrome associated with COVID-19 is reviewed.

Abbreviations: SARS-CoV-2: Severe Acute Respiratory Syndrome Coronavirus 2; M: Membrane; E: Envelope; N: Nucleocapsid; S: Spike; PRRs: Pathogen-Recognition Receptors; PAMPs: Pathogen-Associated Molecular Patterns; STAT: Signal Transducer and Activator of Transcription; JAK: Janus Kinase; MAPK: Mitogen-Activated Protein Kinase; FDA: Food and Drug Administration; IL-6R: IL-6 Receptors; KEGG: Kyoto Encyclopedia of Genes and Genomes

\section{Introduction}

Since the first case of the novel Coronavirus Disease 2019 (COVID-19) was announced in December 2019 in Wuhan, Hubei, China and the first death reported in the United States in February 2020, the disease has produced an astounding death toll, caused incalculable suffering, and destabilized the global economy. Moreover, the pandemic has also shed light on health disparities that exist largely demarcated along racial and socioeconomic lines $[1,2]$. The severe acute respiratory syndrome coronavirus 2 (SARSCoV-2) is the viral pathogenic agent responsible for COVID-19. SARS-CoV-2 is similar in appearance and genetic composition to SARS-CoV-1 (severe acute respiratory syndrome coronavirus 1) and MERS-CoV (Middle East respiratory syndrome coronavirus) with its spherical morphology and crown-like spikes protruding from the envelope surface [3]. SARS-CoV-2 is an RNA virus containing sense strand RNA. SARS-CoV-2 contains a variety of accessory proteins that aid in biosynthesis, maturation, and evasion, however, four structural proteins dominant most illustrations of the virus: membrane (M), envelope (E), nucleocapsid $(\mathrm{N})$, and the prominent spike (S) proteins $[4,5]$.

SARS-CoV-2 has shown the ability to infect and kill men, women, children, and the elderly, however, according to copious amounts of data from around the world, the virus predominantly affects humans over the age of 65 with pre-existing conditions [68]. COVID-19 is a disease characterized by many symptoms and physiological trauma including respiratory distress, fever, muscle paralysis, gastrointestinal illness, chest pain, and other flu-like symptoms [9-11]. Some patients have even reported a loss of smell and nausea associated with the disease. Interestingly, for reasons 
that are still not understood, symptom presentation can be mild to severe. Further, there are many patients who are completely asymptomatic. Research suggests that the virus can be transmitted from person-to-person through direct contact via contaminated surfaces (e.g., fomites), respiratory droplet transmission, and aerosol transmission $[12,13]$.

Aerosol transmission, unlike respiratory transmission, is extremely problematic. Aerosolized microdroplets are ultramicroscopic infectious particles that can remain suspended in the air for long periods (e.g., hours) and travel longer distances compared to the heavier infectious respiratory droplets. Additional transmission mechanisms of the virus are likely to be found in the near future which will likely generate enhancements to existing public health countermeasures to further mitigate transmission. Not surprisingly, in early 2020, the scientific community turned its single focus into developing an effective vaccine. To date, there are over 100 vaccines in the pipeline around the world. Many vaccine candidates are now in the final stages of human clinical trials and therefore answers regarding potency of humoral and cell-mediated immunity will soon be addressed.

\section{SARS CoV-2 Infections and Hyperinflammation}

Innate immunity, the first line of viral pathogen defense, is mediated via pathogen-recognition receptors (PRRs) and viral pathogen-associated molecular patterns (PAMPs). During a typical infection, following PRRs-PAMPs interaction, inflammatory cells and cytokines are regulated and recede once the infection has been controlled by innate and adaptive immune mechanisms. However, for reasons that are unclear, SARS CoV-2 initiates an unrestrained migration of immune cells to the respiratory system and subsequent aggressive release of cytokines and influx of other factors which leads to respiratory dysfunction and destruction [14]. The findings associated with hyperinflammation in COVID-19 patients are consistent with similar coronavirus-related diseases in the past including the severe acute respiratory syndrome and Middle East respiratory syndrome. For both SARS and MERS mortality was significantly attributed to respiratory dysfunction caused by a cytokine storm or hypercytokinaemia $[15,16]$.

Since this condition was first observed in China, the global research community has provided insight into the cytokines that are involved. Specifically, several studies have characterized the prominent pro-inflammatory mediators that contribute to the cytokine storm. These mediators include interleukins (IL6, IL-1, IL-2), chemokines, monokines, tumor necrosis factor alpha (TNF $\alpha$ ), interferons (IFN $\gamma$ ), and many other mediators of inflammation which are highly elevated in the blood during the immune dysregulation event $[17,18]$. These inflammatory response molecules are secreted from a number of immune cells including macrophages, lymphocytes, neutrophils, and natural killer cells $[15,17]$. Remediation strategies to suppress the cytokine storm involve the direct blocking of a particular cytokine or the suppression of distinct signal transduction pathways that facilitate an immune response. Signal transduction pathways are essential intracellular communication complexes that are initiated via ligand-receptor interactions.

Binding of a specific ligand to a cognate receptor at the cell surface results in intracellular signaling processes that lead to the expression of response genes designed to alter cell behavior. At this stage in the pandemic, the research literature regarding the signal transduction pathways that contribute to COVID-19's cytokine storm is limited. The Janus kinase (JAK) and signal transducer and activator of transcription (STAT) pathway and the mitogen-activated protein kinase (MAPK) pathway have been implicated as major signaling pathways that promote hyperinflammation in pathogencompromised patients. The JAK-STAT pathway is known to regulate normal physiologic processes such as apoptosis, differentiation, and proliferation. The JAK-STAT pathway has also been shown to play roles in human cancer and other diseases [19] and mediate functional responses of pro-inflammatory cytokines including IL-6, GM-CSF (granulocyte-macrophage colony-stimulating factor), IL15, G-CSF (granulocyte colony-stimulating factor), and IFN $\gamma$ [20].

\section{Targeting the Cytokine Storm}

JAK-STAT inhibitors were some of the initial therapies that were considered to address the cytokine storm problem observed in COVID-19 patients. Specifically, in the United States it was first thought that the repurposing of FDA (Food and Drug Administration) approved drugs would provide the quickest impact during the earliest stages of the pandemic. According to in silico investigations, several FDA-approved drugs (for non-COVID-19 diseases) have been the subject of intense experimental and clinical evaluation. A considerable amount of attention has been given to antirheumatic drugs (e.g., Baricitinib, Ruxolitinib) for their ability to effectively abrogate proteins in the JAK-STAT pathway and ameliorate inflammatory responses. Ruxolitinib, the Janus kinase $1 / 2$ inhibitor, was recently explored as a way to calm the cytokine storm [21,22]. In a pilot study that occurred several months ago, severe COVID-19 patients who demonstrated hyperinflammation were treated with ruxolitinib. While the sample size was very small, the overwhelming majority of the patients showed clinical improvement as a result of the treatment with ruxolitinib [21].

Baricitinib inhibits both JAK1 and JAK2 and therefore like ruxolitinib blocks cytokine and growth factor signaling pathways. In addition to disrupting JAK-STAT signaling, Baricitinib may also have the capacity to block coronavirus entry into the intracellular environment [23]. There are several ongoing studies seeking to address the synergistic effects of combining cytokine storm modulating drugs. Tocilizumab is a monoclonal antibody directed 
against IL-6 receptors (IL-6R). Binding of the anti-IL-6R antibody to the interleukin- 6 receptor prevents IL-6 from forming the requisite ligand-receptor complex. Tocilizumab has been shown to bind to both the membrane form of the IL-6R and the soluble form of the IL-6R $[24,25]$. In the presence of tocilizumab downstream signaling components are inactive and thus the JAKs and STATs associated with IL- 6 are not activated and gene expression of IL- 6 response genes is suppressed. In a small clinical study (15 patients) tocilizumab treatment produced positive clinical outcomes in some patients and demonstrated an overall decrease in IL- 6 levels in the blood [26].

A slightly larger study consisting of 100 patients provided additional evidence of the effectiveness of tocilizumab in treating severe COVID-19 patients [27]. Despite the reported effectiveness of intravenous tocilizumab administration, the precise biomolecular mechanisms that underpin its positive clinical action is not fully understood and requires more clinical and biochemical research. A particularly interesting bioinformatics study performed by Chen et al. examined the potential impact of a curcumin, glycyrrhizic acid, and vitamin $\mathrm{C}$ dietary regimen on quieting the cytokine storm [28]. Cytoscape, Kyoto encyclopedia of genes and genomes (KEGG), and the Database for annotation, visualization, and integrated discovery (DAVID) were employed to identify putative target genes affected by the nutritional treatment strategy. Biocomputational results demonstrated that many pro-inflammatory signaling pathways linked to the cytokine storm may be blocked or affected by curcumin, glycyrrhizic acid, and vitamin C.

\section{Conclusion}

COVID-19 has had a crippling impact on global institutions. As the worldwide fight rages on for an effective vaccine to fight the virus and create long-term immunity, scientists are looking for other ways to control virus-related symptoms and reduce the length of hospitalizations. For over thirty years immunologists, virologists, and the medical community have known that inflammation plays a critical and protective role during viral challenge. Particularly, members of the tumor necrosis factor and interferon family play distinct roles in mediating an antiviral state by blocking viral replication [29]. Hyperinflammation events have been shown to be responsible for respiratory tissue necrosis, organ failure, and death in severe COVID-19, SARS, and MERS patients [30,31]. In addition to the therapeutic molecules discussed in this review, Sallenave and Guillot reported on methylprednisolone, siltuximab, sarilumab, and many other compounds for their efficacy in suppressing the deadly cytokine storm.

Carefully planned and executed microarray or next generation RNA sequencing studies need to be performed to more intricately investigate the COVID-19-induced cytokine storm and other aspects of COVID-19 such as cell entry, virion production, and virion release. These types of studies will elucidate the molecular mechanisms that play a role in the initiation of hyperactive innate immunity mechanisms and possible control and treatment solutions. Moreover, since this disease has affected over 200 countries around the world, the fight against COVID-19 from a research perspective should involve a diverse number of nations, races, and ethnic groups. It is the contention of the author that demographic groups who are the hardest hit by the devastation caused by the coronavirus (e.g., African Americans), and who are historically underrepresented in national research laboratories may add a unique perspective and attack the virus driven by intrinsic motivation. Lastly, additional exploration of the signal transduction pathways that play a role in cytokine production during SARS-CoV-2 infections will lead to more effective patient treatment strategies.

\section{Acknowledgement}

This work was supported by a grant funded by the National Science Foundation (HRD-1533536).

\section{References}

1. Laurencin C, Mc Clinton A (2020) The COVID-19 pandemic: A call to action to identify and address racial and ethnic disparities. Journal of Racial and Ethnic Health Disparities 7(3): 398-402.

2. Dorn A, Cooney R, Sabin M (2020) COVID-19 exacerbating inequalities in the US. Lancet 395(10232): 1243-1244.

3. Malik Y (2020) Properties of coronavirus and SARS-CoV-2. Malaysian Journal of Pathology 42(1): 3-11.

4. Astuti I, Ysrafil (2020) Severe acute respiratory syndrome coronavirus 2 (SARS-CoV-2): An overview of viral structure and host response. Diabetes \& Metabolic Syndrome 14(4): 407-412.

5. Pillay T (2020) Gene of the month: The 2019-nCoV/SARS-CoV-2 novel coronavirus spike protein. Journal of Clinical Pathology 73(7): 1-4.

6. Lu X, Zhang L, Du H, Zhang J, Li Y, et al. (2020) SARS-CoV-2 infection in children. The New England Journal of Medicine 382(17): 1663-1665.

7. She J, Liu L, Liu W (2020) COVID-19 epidemic: Disease characteristics in children. Journal of Medical Virology 92(7): 747-754.

8. Rothan H, Byrareddy S (2020) The epidemiology and pathogenesis of coronavirus disease (COVID-19) outbreak. Journal of Autoimmunity 109: 102433

9. Ge H, Wang X, Yuan X, Xiao G, Wang C, et al. (2020) The epidemiology and clinical information about COVID-19. European Journal of Clinical Microbiology \& Infectious Diseases 14(1): 1-9.

10. Pascarella G, Strumia A, Piliego C, Bruno F, Del Buono R, et al. (2020) COVID-19 diagnosis and management: A comprehensive review. Journal of Internal Medicine 288(2): 192-206.

11. Zhou Z, Zhao N, Shu Y, Han S, Chen B, et al. (2020) Effect of gastrointestinal symptoms in patients with COVID-19. Gastroenterology 158(8): 22942297.

12. Anderson E, Turnham P, Griffin J, Clarke C (2020) Consideration of the aerosol transmission for COVID-19 and public health. Risk Analysis 40(5): 902-907.

13. Godri Pollitt K, Peccia J, Ko A, Kaminski N, Dela Cruz C, et al. (2020) COVID-19 vulnerability: The potential impact of genetic susceptibility and airborne transmission. Human Genomics 14(1): 17.

14. Coperchini F, Chiovato L, Croce L, Magri F, Rotondi M (2020) The cytokine storm in COVID-19: An overview of the involvement of the chemokine/ 
chemokine-receptor system. Cytokine \& Growth Factor Reviews 53: 2532 .

15. Ye Q Wang B, Mao J (2020) The pathogenesis and treatment of the 'cytokine storm' in COVID-19. The Journal of Infection 80(6): 607-613.

16. Huang C, Wang Y, Li X, Ren L, Zhao J, et al. (2020) Clinical features of patients infected with 2019 novel coronavirus in Wuhan, China. Lancet 395(10223): 497-506

17. Ragab D, Eldin H, Taeimah M, Khattab R, Salem R (2020) The COVID-19 cytokine storm; What we know so far. Frontiers in Immunology 11: 1446.

18. Mangalmurti N, Hunter C (2020) Cytokine storms: Understanding COVID-19. Immunity 53(1): 19-25

19. Flowers L (2013) Targeting JAK-STAT signal transduction pathways in human carcinomas. International Journal of Biosciences 3(8): 241-250.

20. Kiu H, Nicholson S (2012) Biology and significance of the JAK/STAT signalling pathways. Growth Factors 30(2): 88-106.

21. La Rosée F, Bremer H, Gehrke I, Kehr A, Hochhaus A, et al. (2020) The Janus kinase $1 / 2$ inhibitor ruxolitinib in COVID-19 with severe systemic hyperinflammation. Leukemia 34(7): 1805-1815.

22. Luo W, Li Y, Jiang LJ, Chen Q, Wang T, et al. (2020) Targeting JAK-STAT signaling to control cytokine release syndrome in COVID-19. Trends in Pharmacological Sciences 41(8): 531-543.

23. Zhang X, Zhang Y, Qiao W, Zhang J, Qi Z (2020) Baricitinib, a drug with potential effect to prevent SARS-COV-2 from entering target cells and control cytokine storm induced by COVID-19. International Journal of Immunopharmacology 86: 106749.

24. Zhang C, Wu Z, Li JW, Zhao H, Wang GQ (2020) Cytokine release syndrome in severe COVID-19: Interleukin-6 receptor antagonist tocilizumab may

ISSN: 2574-1241

DOI: $10.26717 /$ BJSTR.2020.29.004833

Lawrence 0 Flowers. Biomed J Sci \& Tech Res

(C) This work is licensed under Creative

Submission Link: https://biomedres.us/submit-manuscript.php be the key to reduce mortality. International Journal of Antimicrobial Agents 55(5): 105954.

25. Allegra A, Di Gioacchino M, Tonacci A, Musolino C, Gangemi S (2020) Immunopathology of SARS-CoV-2 infection: Immune cells and mediators, prognostic factors, and immune-therapeutic implications. International Journal of Molecular Sciences 21(13): 4782.

26. Luo P, Liu Y, Qiu L, Liu X, Liu D, et al. (2020) Tocilizumab treatment in COVID-19: A single center experience. Journal of Medical Virology 92(7): 814-818.

27. Toniati P, Piva S, Cattalini M, Garrafa E, Regola F, et al. (2020) Tocilizumab for the treatment of severe COVID-19 pneumonia with hyperinflammatory syndrome and acute respiratory failure: A single center study of 100 patients in Brescia, Italy. Autoimmunity Reviews 19(7): 102568

28. Chen L, Hu C, Hood M, Zhang X, Zhang L, et al. (2020) A novel combination of vitamin $\mathrm{C}$, curcumin and glycyrrhizic acid potentially regulates immune and inflammatory response associated with coronavirus infections: A perspective from system biology analysis. Nutrients 12(4): 1193.

29. Hughes T, Kaspar T, Coppenhaver D (1988) Synergy of antiviral actions of TNF and IFN-gamma: Evidence for a major role of TNF-induced IFNbeta. Antiviral Research 10(1-3): 1-9.

30. Sallenave JM, Guillot L (2020) Innate immune signaling and proteolytic pathways in the resolution or exacerbation of SARS-CoV-2 in Covid-19: Key therapeutic targets? Frontiers in Immunology 11: 1229

31. Liang Y, Wang ML, Chien CS, Yarmishyn A, Yang YP, et al. (2020) Highlight of immune pathogenic response and hematopathologic effect in SARSCoV, MERS-CoV, and SARS-Cov-2 infection. Frontiers in Immunology 11: 1022.

$\begin{array}{ll}\text { BIOMEDICAL } & \text { Assets of Publishing with us } \\ \text { RESEARCHES } & \text { - Global archiving of articles } \\ \text { - Immediate, unrestricted online access } & \text { - Rigorous Peer Review Process } \\ & \text { - Anthors Retain Copyrights } \\ & \end{array}$

